Mathématiques et sciences humaines
Mathematics and social sciences

153 | Printemps 2001

Varia

\title{
Christian Huygens et la table de mortalité de Graunt
}

Christian Huygens and the John Graunt's life's tables

Roland Pressat

\section{OpenEdition}

12 Journals

Édition électronique

URL : http://journals.openedition.org/msh/2855

DOI : $10.4000 / \mathrm{msh} .2855$

ISSN : 1950-6821

Éditeur

Centre d'analyse et de mathématique sociales de l'EHESS

Édition imprimée

Date de publication : 1 mars 2001

ISSN : 0987-6936

Référence électronique

Roland Pressat, "Christian Huygens et la table de mortalité de Graunt », Mathématiques et sciences humaines [En ligne], 153 | Printemps 2001, mis en ligne le 10 février 2006, consulté le 23 juillet 2020 URL : http://journals.openedition.org/msh/2855 ; DOI : https://doi.org/10.4000/msh.2855

〔c École des hautes études en sciences sociales 


\title{
CHRISTIAN HUYGENS ET LA TABLE DE MORTALITÉ DE GRAUNT
}

\author{
Roland PRESSAT ${ }^{1}$
}

\begin{abstract}
RÉSUMÉ - En analysant le contenu de la table de mortalité de Graunt, on peut mettre en relief le côté factice de certaines différences établies par les frères Huygens telles que les présentent Jacques Véron et Jean-Marc Rohrbasser (Math. et Sci. hum., $n^{\circ} 149$, p. 7-21). Christian Huygens en calculant la survie du dernier décédé d'un groupe de deux personnes a fait apparaître les notions de probabilité conditionnelle et d'espérance de vie conditionnelle, notions qui ne sont pas dégagées dans l'article cité.
\end{abstract}

MOTS-CLÉS - Mortalité, Espérance de vie, Probabilité conditionnelle.

SUMMARY- Christian Huygens and the John Graunt's life's tables

By analysing the John Graunt's life's tables matters, it is possible to point out the somehow artificial presentation way of some discrepancies established by the Huyghens brothers as reported by Jacques Véron et Jean-Marc Rohrbasser (Math. et Sci. Hum., $n^{\circ} 149$, p. 7-21). By computing the life's span of the last surviving, out of a group of two persons, Christian Huygens has established the notion of conditional probability as well as the notion of the conditional life's expectancy, both notions which have not explained, neither mentioned in the quoted article.

KEYWORDS - Mortality, Life expectancy, Conditional probability.

On crédite Christian Huygens d'être à l'origine de la notion d'espérance mathématique. Selon P. Deheuvels ${ }^{2}$ «le nom d'espérance (...) apparaît en latin sous le nom de expectatio dans le traité de calcul des probabilités de cet auteur avec l'interprétation d'être le juste prix auquel un joueur accepterait de céder sa place dans une partie ».

Dans l'optique d'alors, où le calcul des probabilités naissant s'attachait donc à résoudre des problèmes posés par les jeux de hasard, Huygens va transposer cette notion d'espérance mathématique dans le cadre de cette loterie qu'est notre durée d'existence, débouchant sur ce que nous appelons l'espérance de vie ${ }^{3}$. Plus précisément Huygens verra dans la série des décès $\{d(x, x+a)\}$ d'une table de mortalité la donnée de la loi de probabilité qui gouverne notre durée de vie ; mais l'espérance de vie qui en découle ne saurait être qu'un indice synthétique et, en aucun cas, une probabilité et elle ne saurait donc intervenir quand il s'agira de parier sur la survie d'une personne à un âge $x$

\footnotetext{
${ }^{1}$ Institut National d'Études Démographiques, 133, boulevard Davout, 75980 Paris Cedex 20.

2 Paul Deheuvels, La probabilité le hasard et la certitude, Paris, Presses Universitaires de France (coll.

«Que sais-je ? »), 1982.

3 Voir Rohrbasser et Véron, « Les frères Huygens et le 'calcul des aages' », Population 54 (6), 1999.
} 
donné. C'est pourtant ce que croit son frère Louis. Et une discussion s'instaure quand Louis constatant que l'espérance de vie à 6 ans et à 16 ans, selon la table de Graunt, est sensiblement égale à 20 ans, il en conclut que « la partie est environ esgale lors qu'on gage qu'une personne de 6 . ou une de 16 . vivront encor 20 ans ».

Louis confond espérance de vie (c'est-à-dire survie moyenne) et survie probable. Pour détromper son frère, Christian avait deux options possibles.

1) Montrer qu'avec un pari sur une survie de 20 ans la partie n'est pas égale et qu'il convient de gager 25 contre 39 à 6 ans et 26 contre 39 à 16 ans : c'est l'option choisie par Christian.

2) Établir que la partie est égale si l'on retient comme enjeux du pari les survies probables à 6 et 16 ans. Le recours à une lecture - nécessairement approximative - sur la courbe de survie conduit respectivement aux valeurs, assurant un pari équitable, de 14,8 ans et 15,0 ans (Figure 1). Si Christian Huygens, qui avait eu recours à une telle lecture graphique pour relativiser la signification de la vie moyenne $(18,22$ ans selon la table de Graunt) en lui opposant une vie probable d'environ 11 ans, n'a pas adopté la même conduite dans le cas présent, c'est probablement qu'il voulait marquer de la meilleure manière combien des joueurs «se feroient tort en acceptant moins de 20 ans assurez $\gg$.

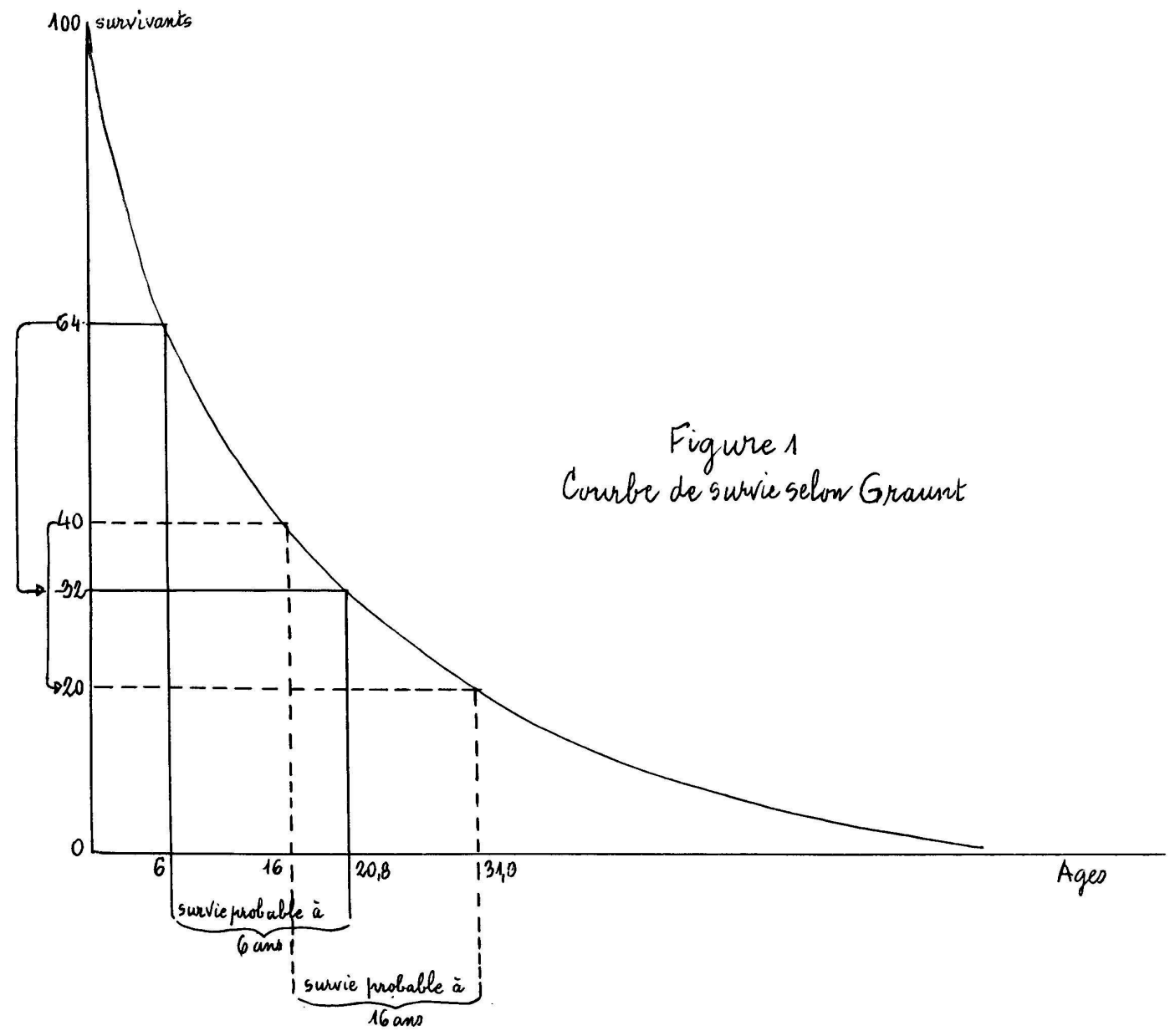

Figure 1. Courbe de survie selon Graunt 
Sans doute l'objet principal des calculs de Christian est-il de montrer la distance qu'il y a entre les termes du pari que propose Louis et ce que montre son raisonnement rigoureux. Mais soucieux de mettre en valeur cette rigueur il insiste aussi sur le fait qu' «il est un peu plus apparent pour un de 16 ans que pour un de 6 de vivre encore 20 ans ». Or l'écart est très faible entre 25 contre 39 et 26 contre 39 , comme est très faible celui existant entre les survies probables de 14,8 ans et 15,0 ans. Et, de son côté, Louis peut arguer qu'entre $e(6)=20,78$ ans et $e(16)=20,25$ ans l'écart est bien mince (nous notons $e(x)$, l'espérance de vie à l'âge $x$ et, plus loin, $s(x)$ la fonction survie à l'âge $x$ ). Il est alors piquant de mettre en lumière ce qui se cache derrière ces quasisimilitudes.

Retournons pour cela à la table de Graunt. On sait qu'il s'agit d'une construction largement théorique Graunt lui-même affirmant qu'il comble le vide séparant $s(6)=64$ de $s(76)=1$ en recherchant «six nombres moyens proportionnels entre 64 le nombre de survivants à 6 ans, et 1 , celui qui survit à 76 ans ». Cette recherche des «nombres moyens proportionnels » a débouché, dans les faits, sur l'adoption de quotients de mortalité décennaux constants et égaux à $\frac{3}{8}$. Voici ce qu'il en est lorsque l'on compare la table de Graunt au-delà de 6 ans à une table construite avec de tels quotients de mortalité :

\begin{tabular}{|c|c|c|}
\hline & \multicolumn{2}{|c|}{ Survivants selon la table } \\
\hline Age & de Graunt & à quotients constants \\
\hline 6 & 64 & 64 \\
16 & 40 & 40 \\
26 & 25 & 25 \\
36 & 16 & 15,625 \\
46 & 10 & 9,77 \\
56 & 6 & 6,10 \\
66 & 3 & 3,81 \\
76 & 1 & 2,38 \\
\hline
\end{tabular}

Aux arrondis près l'accord est parfait jusqu'à 56 ans.

Si Christian Huygens avait travaillé avec des données à quotients strictement constants il aurait conclu qu'il était aussi « apparent pour un de 16 ans que pour un de 6 de vivre encore 20 ans » car on a bien rigoureusement

$$
\frac{s(26)}{d(6,26)}=\frac{25}{39}=\frac{s(36)}{d(16,36)}=\frac{15,625}{24,375}=0,641
$$

Ce sont bien évidemment les modalités de la décroissance de la fonction survie $s(x)$ qui sont en cause. Au simple examen de la courbe représentative de cette fonction (voir figure 1), l'idée d'une décroissance exponentielle apparaît, formalisée par 
l'écriture $s(x)=e^{\square a x+b}, a>0$. Cette idée est appuyée par le fait que, précisément, une décroissance à quotients constants est la caractéristique d'une fonction survie exponentielle.

En pareil cas on a :

- pour l'espérance de vie à l'âge $x: e(x)=\frac{1}{a}$;

- pour le rapport des chances à l'âge $x$ de dépasser $x+k: \frac{1}{e^{a k} \square 1}$;

- $\quad$ pour la survie probable à l'âge $x: \frac{1 \mathrm{n} 2}{a}$.

Ainsi ces trois quantités, indépendantes de $x$, sont donc constantes (en appliquant ces formules on trouve respectivement 21,$28 ; 0,641 ; 14,75$ ans) et il n'est pas loin d'en être ainsi avec les données de la table de Graunt dans le secteur de celle-ci où les quotients de mortalité restent à peu près constants. On a confirmation de ce fait en constatant graphiquement combien sont faibles les variations de l'espérance de vie avec l'âge entre 6 et 36 ans. (Voir Rohrbasser et Véron, op. cit., p. 1009).

Cette particularité de la courbe de survie de Graunt n'a pas retenu l'attention de Christian Huygens. Qu'aurait-il bien pu dire s'il avait pu observer que les termes d'un pari à durée de survie donnée ne variaient pas avec l'âge ?

Pour conclure, faisons référence à un texte ambigu de Rohrbasser et Véron ${ }^{4}$ où ces auteurs opposent une différence faible à une différence certaine :

«Pour Lodewijk, la différence est faible, mais pour Christian dont le raisonnement est probabiliste, cet argument d'une quasi-égalité n'est pas fondé, puisque les données montrent qu'il est « certain » qu'une différence existe ».

Ce paragraphe arrive après la présentation de l'inégalité des chances d'une survie de 20 ans à 6 et 16 ans selon Christian. On ne comprend pas ce que Rohrbasser et Véron veulent dire : s'agit-il, concernant Louis, de la différence qu'il a mise en lumière à partir de sa référence erronée à e (6) et e (16), ou de celle que Christian lui fait constater à partir de ses rapports de chances ? Chacun parle-t-il pour son propre compte ou entre-til dans les vues de son partenaire pour les contredire ? ${ }^{5}$ Quelle que soit la réponse, nos développements devraient mettre nos deux frères d'accord sur un point : dans les deux approches de la survie à 6 et 16 ans, les différences constatées à ces âges sont insignifiantes, découlent du mode de construction de la table de Graunt et relèvent, en dernier ressort, d'un problème d'arrondis...

Dans leur texte de Population Rohrbasser et Véron se sont attachés à dégager l'esprit probabiliste qui préside à la présentation, par Christian Huygens, de la table de mortalité de Graunt. On aurait mieux compris ce qui distingue la démarche intellectuelle de Louis Huygens, lorsqu'il calcule une vie moyenne, de celle de Christian quand, à

\footnotetext{
${ }^{4}$ Op.cit. p. 1001 ou encore, des mêmes auteurs, Mathématiques et Sciences humaines, $\mathrm{n}^{\circ}$ 149, 2000, p. 17.

${ }_{5}^{5}$ Il semble bien, d'après une citation suffisamment étoffée de Louis, que rapporte Dupâquier, (Cf. L'invention de la table de mortalité), et qui manque dans Rohrbasser et Véron, que Louis fasse référence à ses propres calculs.
} 
partir des mêmes calculs, ce dernier parle d'espérance de vie, si une interprétation correcte du tableau 5 de leur article de Mathématiques et Sciences humaines ( ${ }^{\circ} 149$, 2000, p. 17) - qui n'est que la reprise de ce qui est dit dans Population - avait été proposée. Or en écrivant «Quand Christian fait cette opération [en l'occurrence la multiplication de 36 chances de décès entre 0 et 6 ans par l'âge médian 3 ans], c'est pour calculer les chances de vivre 3 années » Rohrbasser et Véron se méprennent : ces «chances de vivre » sont données directement par les 36 décès et leur produit par 3 qui s'exprime en années n'a pas une signification probabiliste mais fait état de l'apport des 31 chances en termes d'années de vie.

Maladroits, donc, dans cette présentation de la pensée probabiliste de Christian Huygens, les auteurs n'ont pas su analyser pleinement la richesse de cette dernière : avec leurs calculs du tableau 6 de Mathématiques et Sciences humaines ( $\mathrm{n}^{\circ} 149,2000$, p. 20) (repris du tableau 6 erroné de Population et qui a fait l'objet de rectifications par nos soins (une d'entre elles a été omise à la première ligne où l'on doit lire 20,8 au lieu de 20,3)), ils en sont restés au niveau du pur calcul de la survie du dernier décédé d'un groupe de deux personnes.

Cette joliesse qui consiste à présenter la démarche de Christian pour résoudre ce dernier problème, « sous la forme d'un arbre » laisse dans l'ombre les détails d'une telle démarche et, surtout, sa signification profonde, à savoir l'introduction de ces notions, alors toutes neuves, de probabilité conditionnelle et d'espérance mathématique conditionnelle.

Christian Huygens s'est donc attaqué à la question de la survie au sein d'un groupe de deux personnes. Il ne traitera que le cas simple où les deux personnes ont le même âge (16 ans) lors de la constitution du couple, toujours en se référant à la table de Graunt.

Deux questions surgissent que Huygens va aborder :

- Quelle est l'espérance de vie du premier décédé ?

- Quelle est l'espérance de vie du survivant?

C'est la réponse à cette dernière question qui fait l'objet du fameux tableau 6 . Nous allons reprendre le problème en le traitant avec un peu plus de rigueur que ne l'a fait C. Huygens.

Nous nous référerons à la figure 2 sur laquelle on trouve :

- une échelle d'âges ;

- une échelle de durées écoulées depuis l'âge de 16 ans.

Nous distinguerons les deux membres du couple formé à l'âge de 16 ans par les lettres $A$ et $B$ qui désigneront également leurs durées de survie depuis l'âge de 16 ans et nous noterons $M$ la durée écoulée lors du décès du survivant. 

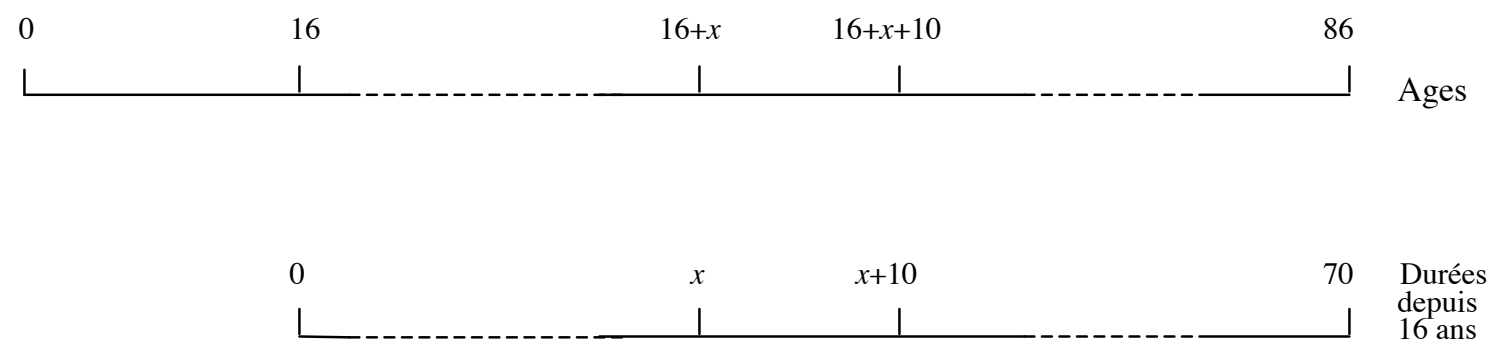

\begin{tabular}{|c|c|c|c|c|c|c|c|c|c|c|c|c|c|c|c|c|c|c|}
\hline 0 & 6 & & 16 & & 26 & & 36 & & 46 & & 56 & & 66 & & 76 & & 86 & Ages \\
\hline 36 & 1 & 24 & 1 & 15 & 1 & 9 & 1 & 6 & 1 & 4 & 1 & 3 & $\perp$ & 2 & 1 & 1 & $\downarrow$ & Décès \\
\hline 0 & 64 & & 40 & & 25 & & 16 & & 10 & & 0 & & 3 & & 1 & & 0 & urviva \\
\hline
\end{tabular}

Figure 2. Échelle d'âges et caractéristiques de la mortalité selon Graunt

Plaçons-nous dans l'hypothèse où $A$ décède dans l'intervalle de durées $(x, x+10)$.

- Tant que le décès de $B$ se situe à une durée inférieure à $x$ c'est la durée lors du décès de $A$ qui détermine la durée lors du décès du survivant soit donc $M=x+5$, valeur moyenne en supposant les décès uniformément répartis entre $x$ et $x+10$. Cette situation a lieu avec la probabilité $\frac{1}{40}[s(16+x) \square s(16+x+10)]$ (le crochet représente les décès qui fixent la probabilité en les rapportant aux 40 survivants à 16 ans).

- Quand $B$ décède entre $x$ et $x+10$ il entre en concurrence avec $A$ pour fixer la durée lors du décès du survivant. On invoquera ici un résultat connu en probabilité géométrique : en choisissant au hasard un couple de points sur un segment de longueur $l$, l'espérance mathématique du segment le plus long ainsi déterminé est égale à $\frac{2 l}{3}$. La moyenne de la durée de survie la plus longue au sein de l'intervalle $(x, x+10) \quad$ vaut $\quad M=x+10 x \frac{2}{3}=x+6,67$ avec la probabilité $\frac{1}{40}[s(16+x) \square s(16+x+10)]$.

- Quand $B$ décède au-delà de $x+10$ le décès du survivant se placera en moyenne à la durée $M=x+10+e(16+x+10)$ ce qui a lieu avec la probabilité $\frac{1}{40} s(16+x+10)$.

On peut résumer tout cela dans le tableau ci-après : 


\begin{tabular}{|c|c|c|c|}
\hline \multicolumn{4}{|c|}{ Loi de probabilité conditionnelle de $M$ avec $x<A<x+10$} \\
\hline & $0<B<x$ & $x<B<x+10$ & $B>x+10$ \\
\hline$M$ & $x+5$ & $x+6,67$ & $x+10+e(16+x+10)$ \\
\hline Probabilité & $\frac{1}{40}[s(16) \square s(16+x)]$ & $\frac{1}{40}[s(16+x) \square s(16+x+10)]$ & $\frac{1}{40} s(16+x+10)$ \\
\hline
\end{tabular}

Nous avons tous les éléments pour calculer la suite des espérances de vie conditionnelles répondant à l'écriture $E\{M / x<A<x+10\}$.

Ainsi, à titre d'exemple, avec $10<A<20$, (éventualité de probabilité égale à 9/40), se trouvent associées :
si $0<B<10, M=15$
avec comme probabilité conditionnelle
$\operatorname{Pr}\{M=15 / 10<A<20\}=15 / 40$
si $10<B<20, M=16,67$ avec comme probabilité conditionnelle
$\operatorname{Pr}\{M=16,67 / 10<A<20\}=9 / 40$
si $B>20, M=37,50$
avec comme probabilité conditionnelle
$\operatorname{Pr}\{M=37,5 / 10<A<20\}=16 / 40$

d'où il résulte pour $M$ comme espérance de vie conditionnelle

$$
E\{M / 10<A<20\}=\frac{1}{40}(15 \square 15+16,67 \square 9+37,5 \square 16)=24,4 \text { ans }
$$

Le mode de calcul de Huygens diffère du nôtre par la façon dont est traitée la question quand $A$ et $B$ sont tous les deux en posture d'être survivants ; Huygens partage alors les «chances » en deux, $4 \frac{1}{2}$ s'appliquant à $A$ en tant que survivant de 15 ans (médiane de l'intervalle 10 - 20), 4 $\frac{1}{2}$ «pour 16, 17, 18, 19, ou 20 ans qui vaut autant que $4 \frac{1}{2}$ pour 18 ans » appliqué à $B$ (pour ce dernier décompte, à la médiane 17,5 ans de l'intervalle 15 - 20 ans, est substituée curieusement la valeur 18 ans). Cette façon d'opérer et la justification qui en est donnée n'emportent pas la conviction, ce qui n'empêche que les résultats sont soit identiques soit égaux à une décimale près (ainsi dans notre exemple 24,3 contre 24,4 ).

On ne saurait dire comme le font Rohrbasser et Véron ${ }^{6}$ que « aujourd'hui, l'espérance de vie a perdu son caractère probabiliste ${ }^{7}$. De plus, les espérances de vie

\footnotetext{
${ }^{6}$ Cf. Population, 55 (4-5), 2000, p. 836.

${ }^{7}$ Peut-être que par cette expression ambiguë ils ont voulu avancer l'idée que l'espérance de vie n'est plus interprétée comme l'espérance mathématique de la loi de probabilité attachée à la série des décès d'une
} 
attachées au survivant d'un couple, présentées de façon neutre par ces auteurs sous l'appellation "durée de vie moyenne », ont un sens probabiliste parce que, en tant qu'espérances de vie conditionnelles, elles sont dépendantes de la loi de probabilité d'un des membres du couple. C'est en appliquant cette loi de probabilité - définie par les quantités $\operatorname{Pr}\{x<A<x+10\}$ - que l'on débouchera sur l'espérance de vie du survivant d'un couple, à la naissance de ce couple :

$$
E\{M\}=\prod_{x=0,10,20, \ldots}^{60} E\{M / x<A<x+10\} . \operatorname{Pr}\{x<A<x+10\}=29,22 \text { ans }
$$

En s'emparant de la table de mortalité de Graunt et en opérant à son sujet un transfert heureux de la notion de pari qui est à l'origine du calcul des probabilités, Christian Huygens a magistralement illustré le concept d'espérance mathématique sous sa forme la plus immédiate et de façon plus ingénieuse encore, comme nous venons de le montrer, sous la forme d'espérance mathématique conditionnelle.

table de mortalité. Rien ne permet de dire cela : la manière d'interpréter une espérance de vie n'est pas une affaire de mode mais répond à la nature des problèmes posés. Au demeurant la vision probabiliste est inhérente à la notion de table; ne parle-t-on pas de probabilités de survie et les anglo-saxons qui n'ont pas adopté notre appellation quotient n'ont-ils pas recours à probability of dying ou death probability? 